\title{
Morphologie der Sinnesepithelien des Vestibularorgans
}

\author{
Kozo Watanuki und A. Meyer zum Gottesberge \\ Innenohr-Forschungslaboratorium der Hals-Nasen-Ohrenklinik \\ der Universität Düsseldorf, Düsseldorf, Bundesrepublik Deutschland
}

\begin{abstract}
Watandki, K. and Meyer zum Gottesberge, A. Morphological Study of the Sensory Epithelium of Vestibular Organs. Tohoku J. exp. Med., 1971, 104(1), 55-63 - For the study of the surface areas of the vestibular sensory epithelia, the silver reaction method and the succinic dehydrogenase staining were successfully used in demonstrating the overall view of each sensory epithelium. (1) In the central part of the sensory epithelium of the crista ampullaris, there were often found larger sensory cells, while in the peripheral regions, smaller sensory cells were found. The cell density was larger in the peripheral regions than in the central part. (2) About two-thirds of all sensory cells in the striola were type I cells. Outside the striola, type I sensory cells were about 45 per cent, while type II cells about 55 per cent of all sensory cells. (3) Larger sensory cells of type I which were found in the striola were usually innervated by common nerve chalices. (4) Larger sensory cells in the striola contained more succinic dehydrogenase activities than smaller cells which locate both in the striola and in other peripheral parts of the macula. ___ sensory epithelia; vestibular organs; silver reaction; succinic dehydrogenase; striola
\end{abstract}

Das Sinnesepithelium der Cirsta ampullaris wird durch die Cupula überdeckt, während sich die Otolithenmembran über die Macula sacculi sowie utriculi ausdehnt. Unter dieser Überdachung bilden die Sinnesepithelien des Vestibularorgans eine einreihige Zellschicht von mosaikartiger Struktur, bestehend aus Sinnes- und Stützzellen. Ungeachtet der scheinbaren Strukturähnlichkeit zeigen sich regionale Unterschiede in der Anordnung.

Retzius (1884) und Iwata (1924) untersuchten erstmalig tierexperimentell die Oberflächenstruktur der Sinnesepithelien des Vestibularorgans. Lindeman (1967, 1969) untersuchte nun phasenkontrastmikroskopisch unter Anwendung der Häutchenpräparation das Sinnesepithelium des Vestibularapparates beim Meerschweinchen und berichtete umfassend darüber. Im folgenden Beitrag findet die Silberfärbung (Watanuki 1968, Watanuki et al. 1969, Katagiri et al. 1968) die kürzlich für die Oberflächenbetrachtung des Vestibularorgans erfolgreich eingesetzt wurde (Watanuki et al.) sowie die Succenodehydrogenasereaktion (Barka und Anderson, 1963) Anwendung.

Received for publication, October 19, 1970.

Director: Prof. A. Meyer zum Gottesberge. 


\section{MethodiK}

Fünfzig Innenohren junger, weisser Meerschweinchen mit einem durchschnittlichen Gewicht von $300 \mathrm{~g}$ wurden $\mathrm{zu}$ diesen Untersuchungen genommen. Nach einer Pentobarbitalnarkose und anschliessender Dekapitation wurden die Felsenbeine entnommen. Nach Entfernung des Stapes wurde die knöcherne Wand des Vestibulums eröffnet und mit einer harten Nadel Sacculus, Utriculus sowie die drei Ampullen des Canalis semicircula. ris freigelegt. Unter Aqua dest. wurden die Wände des Utriculus, Sacculus und der Ampullen eröffnet und für ungefähr eine Minute in eine $0.2 \%$ Silbernitratlösung gegeben. Darauf erfolgte erneut für Sekunden eine Aqua dest. Spülung unter Entfernung der Otolithenmembran und der Cupula. Anschliessend wurde bei Tages- oder Fluoreszenzlicht über 24 Stunden hin in 10\% Formalinlösung fixiert.

Zur Succenodehydrogenasereaktion wurden die Felsenbeine für 30 Minuten bei $37^{\circ} \mathrm{C}$ in folgender Lösung inkubiert:

$\begin{array}{ll}\text { Natriumsuccinat, } 0.06 \mathrm{~m} & 1.0 \mathrm{ml} \\ \text { Nitro BT, 0.2\% } & 2.5 \mathrm{ml} \\ \text { Phosphat-Puffer, 0.2 m (pH 7.4) } & 1.0 \mathrm{ml} \\ \text { Ringerlösung } & 0.5 \mathrm{ml}\end{array}$

Nach 24 stüdiger Formalinfixation wurde das Material für die lichtmikroskopsichen Untersuchungen präpariert.

\section{Ergebnisse}

\section{1) Macula sacculi et utriculi}

Die Sinnesepithelien des Utriculus sind deltaförmig, wobei der vordere Abschnitt breiter als der hintere Teil ist. Die sichelförmigen Sinnesepithelien des Sacculus dehnen sich superior posterior aus (Dorsallappen nach Burlet und Haas 1924, Burlet und Hoffman 1929; Dorsalzipfel nach Werner 1933). Die Sinnesepithelien bilden den konkaven Teil der Macula utriculi und den superior-posterioren Anteil der Macula sacculi. In diesem Abschnitt finden sich anzahlmässig weniger Sinneszellen als in anderen peripheren Zonen (Abb. 3, 4 und 7).

In der Striola sind die Sinneszellen gewöhnlich größer als in anderen peripheren Abschnitten. Es finden sich jedoch zwischen diesen großen Zellen eine geringe Anzahl kleinerer. Die Dichte der Sinnsezellen in der Striola ist geringer als im peripheren Teil. AuBerhalb der Striola sind die Sinnes- und Stuitzzellen radiär angeordnet.

Es ist bekannt, daß die Sinneszellen morphologisch in Richtung der Kinozilien polarisiert sind, dabei von Stereozilienbündeln einseitig umgeben (Löwenstein und Wersäll 1959, Wersäll 1961). Wie Löwenstein et al. (1964) und Flock (1964) zeigten, sind in der Macula sacculi die Sinneszellen von der Striola weg polarisiert, während sie in der Macula utriculi (Sponedlin 1964, 1965) zur Striola hin angeordnet sind. Die Dichte der Sinneszellen ist in der Macula utriculi größer als in der Macula sacculi.

Bei verschiedenen Fokussierungen der Häutchenpräparate des Sinnesepithels unter dem Lichtmikroskop sieht man, daß die Form der einzelnen Sinneszellen von der Basis zur Spitze hin eingehalten wird. Die flaschenförmigen Sinneszellen mit den Nervenkelchen des Typ I und den zylindrischen Zellen des Typ II sind ebenso 


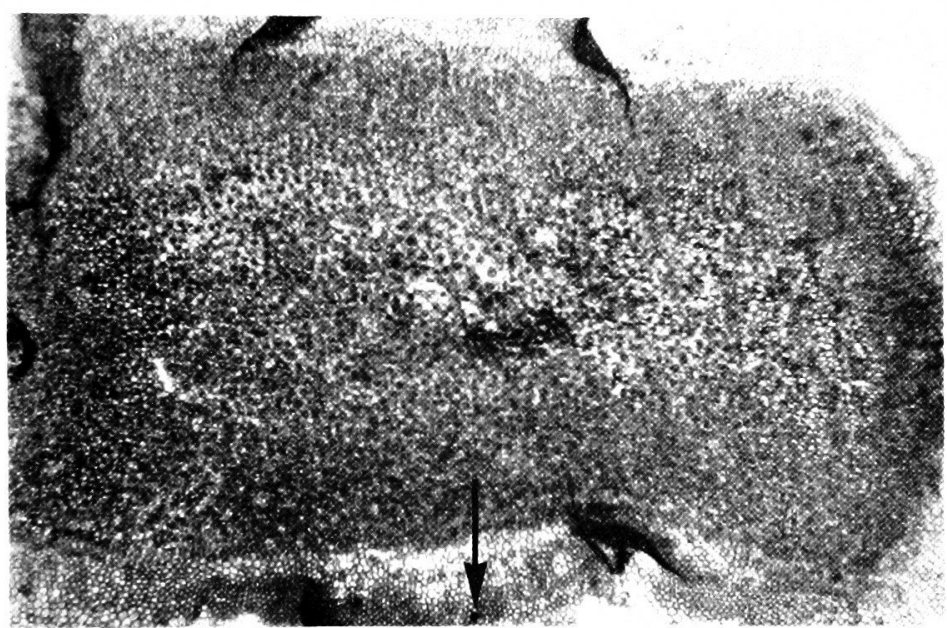

Abb. 1. Übersicht des Sinnesepitheliums der Crista lateralis im rechten Innenohr. Die Oberflächenstruktur ist kontrastreich und klar nach der Silberfärbung. Die Pfeilspitze zeigt die Richtung zum Bogengang hin an. $\times 100$.

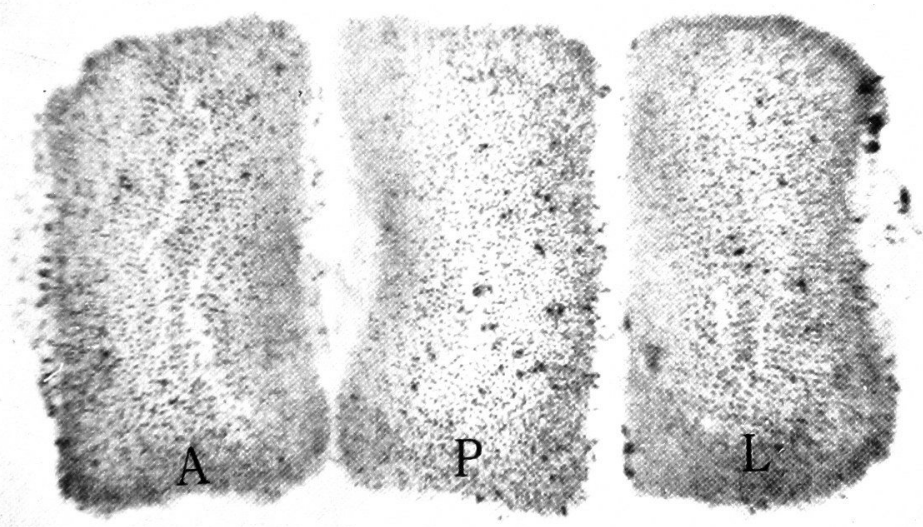

Abb. 2. Drei Sinnesepithelien der Crista ampullaris vom gleichen Innenohr nach der Succinodehydrogenasereaktion. Die einzelne Zelle ist klar sichtbar und zeigt den Unterschied zwischen den zentralen und peripheren Regionen an, in Zellgrösse sowie Dichte. A: Crista anterior. P: Crista posterior. L: Crista lateralis. $\times 40$.

leicht zu identifizieren. In der Striola der Macula sacculi und utriculi werden die meisten Sinneszellen mit großer, freien Oberfläche, umgeben von dem gemeinsamen Nervenkelch, angetroffen. Ab und zu werden auch drei benachbarte Sinneszellen von einem Nervenkelch innerviert. In der Striola der Macula sacculi sowie utriculi setzen sich die Sinneszellen zu zwei Drittel aus dem Typ I zusammen, während im übrigen peripheren Teil das Verhältnis der Sinneszellen des Typ I und Typ II 1,0:1,2 beträgt. Die relative Verteilung der Sinneszellen des Typ 


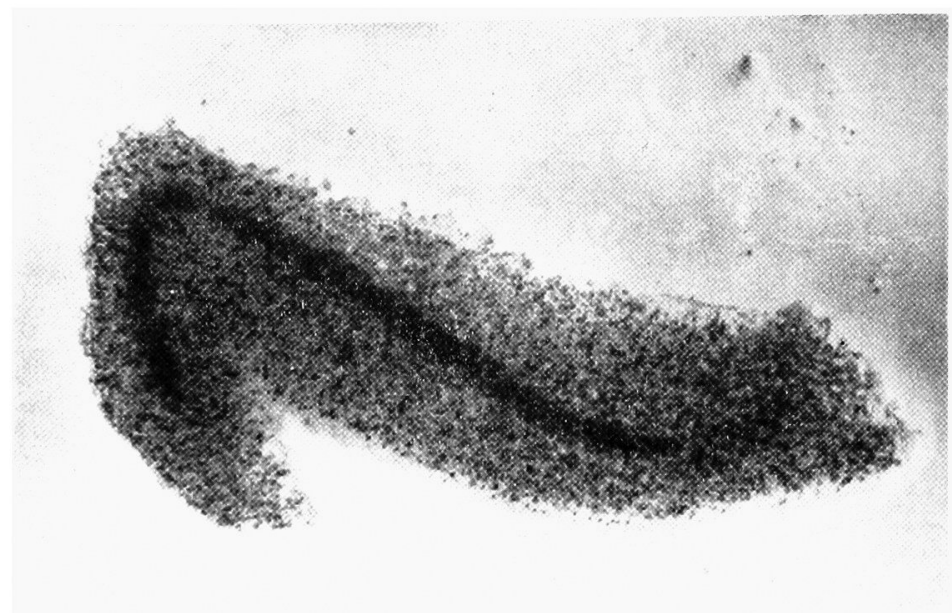

Abb. 3. Sinnesepithel der Macula sacculi nach der Succinodehydrogenasereaktion. Die grossen Zellen der Striola sind deutlich angefärbt und zeigen klar die Lokalisation der Striola an. $\times 60$.

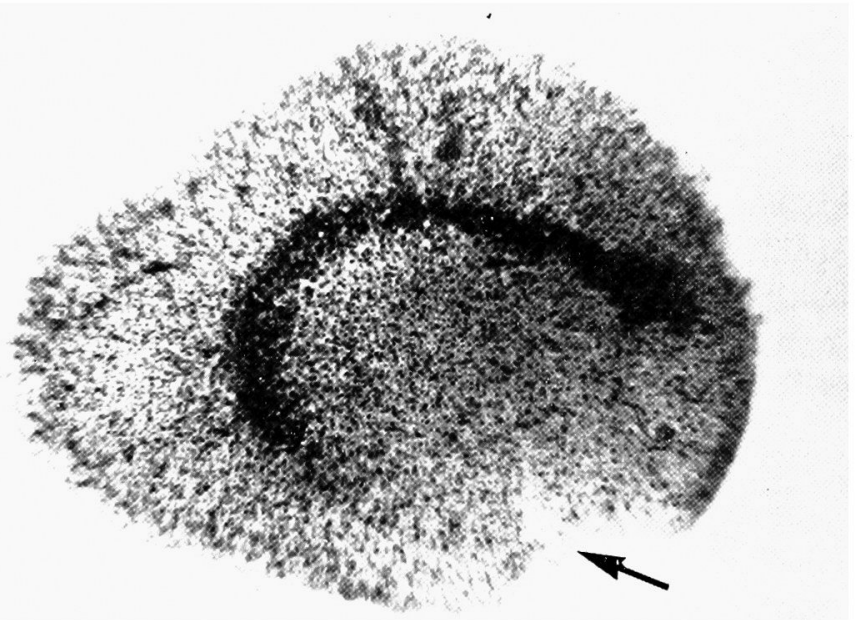

Abb. 4. Das Sinnesepithelium der Macula utriculi nach der Succinodehydrogenasereaktion. In den konkaven Abschnitten des Sinnesepithels (Pfeil) sind die Sinneszellen spärlich verteilt. Die unterschiedliche Färbung der Striola ist klar sichtbar. $\times 60$.

I und Typ II dehnt sich in der Peripherie der Macula nicht so regelmäßig aus, auch zeigen sich von Spezies zu Spezies individuelle Unterschiede. Die Gesamtanzahl der Sinneszellen des Vestibularapparates wird an 10 Tieren dh. 20 Innenohren in der folgenden Tabelle gezeigt.

\section{2) Crista ampullaris}

Das Sinnesepithelium bedeckt sattelförmig die Crista, die senkrecht in Richtung zum Canalis semicircularis liegt. Das Oberflächenmuster der Sinnesepithelien der 


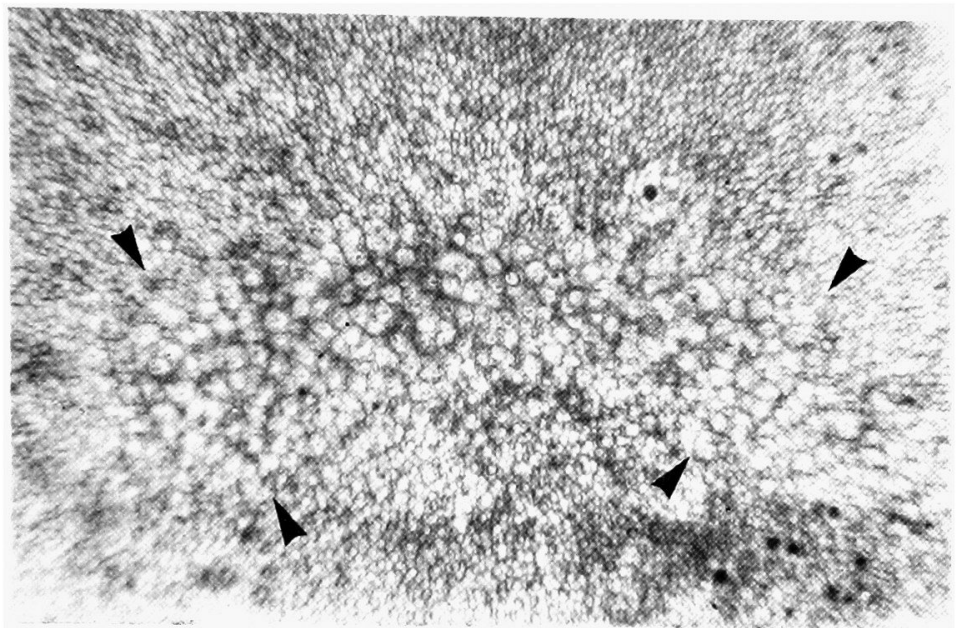

Abb. 5. Oberflächenstruktur der Sinnesepithelien der Macula utriculi nach der Silberfärbung. In der Striola (zwischen den Pfeilen) sind grössere Zellen sichtbar. Ausserhalb sind die Sinnes und Stützzellen strahlenförmig, von der Striola weg, angeordnet. $\times 150$.

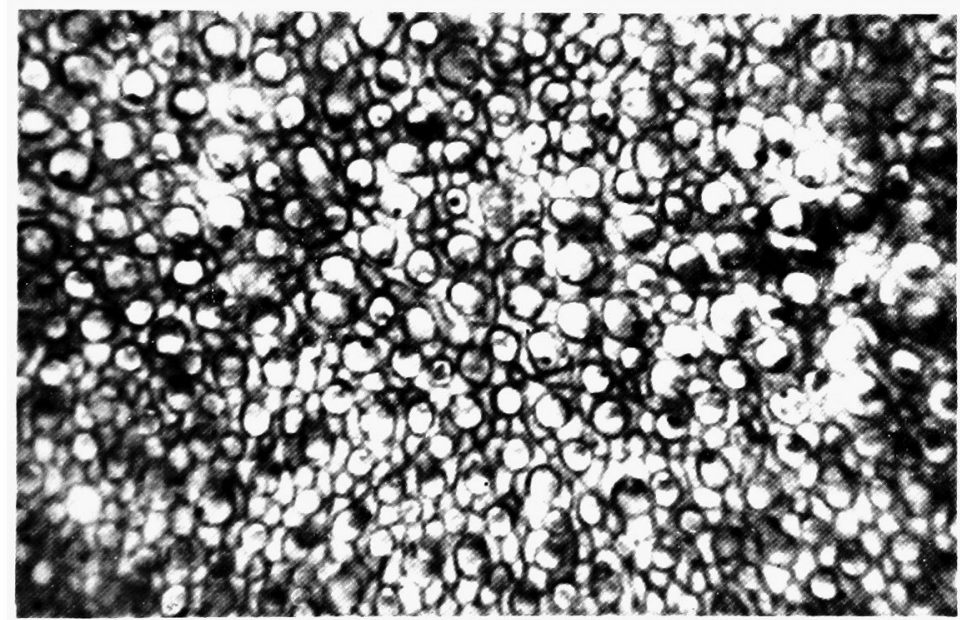

Abb. 6. Eine starke Vergrösserung der Striola in der Macula utriculi. Zwischen den grossen Zellen finden sich eine geringe Anzahl kleinerer Sinneszellen. $\times 600$.

Crista posterior ist rechteckig mit abgerundeten Ecken, während das der Crista anterior und Crista lateralis trapezförmig ist (Abb. 2). Im allgemeinen sind Crista anterior und lateralis in Form und Größe symmetrisch.

An der freien Oberfläche der Epithelien greiften Stütz- und Sinneszellen ineinander eine mosaikartige, kompakte Reticularschicht bildend. Eine Sinneszelle wird von 3-5 Stützzellen umgeben, gewöhnlich sind es vier. Die zentralgelegenen Sinneszellen der Crista sind meistens von größer Oberfläche, die der peripheren Region kleiner und weniger unterschiedlich in Form und Größe. 


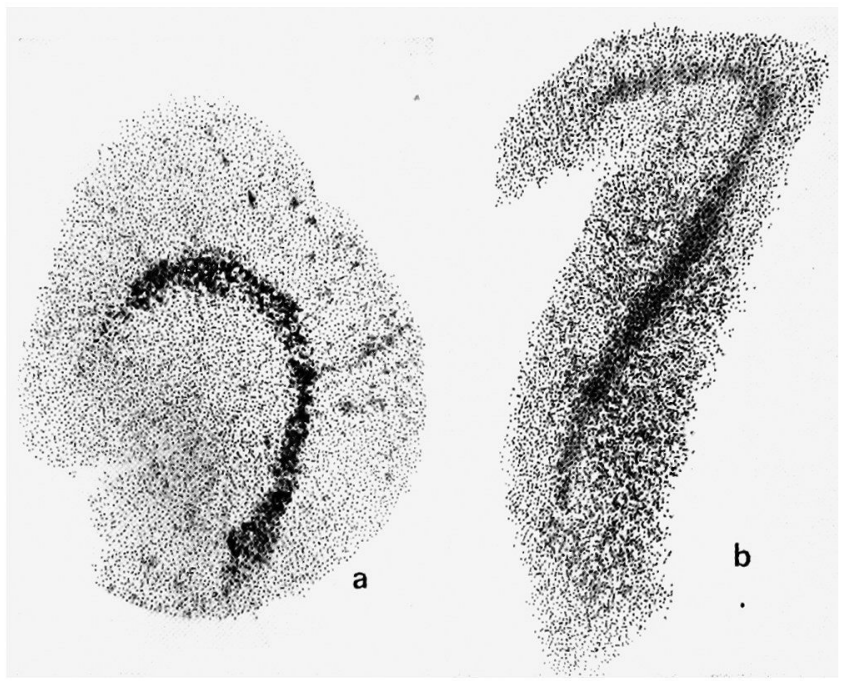

Abb. 7. Eine halbschematische Darstellung der Sinnesepithelien der Macula utriculi (a) und Macula sacculi (b). Diese graphische Darstellung wurde durch exakte Tuschekennzeichnung der Abb. 3. u. 4 angefertigt.

TaBelle 1. Anzahl der Sinneszellen der Sinnesepithelien des Vestibularorgans beim Meerschweinchen

\begin{tabular}{|c|c|c|c|c|c|c|}
\hline \multicolumn{2}{|c|}{ Tier } & $\begin{array}{l}\text { Crista } \\
\text { anterior }\end{array}$ & $\begin{array}{c}\text { Crista } \\
\text { lateralis }\end{array}$ & $\begin{array}{l}\text { Crista } \\
\text { posterior }\end{array}$ & $\begin{array}{l}\text { Macula } \\
\text { sacculi }\end{array}$ & $\begin{array}{l}\text { Macula } \\
\text { utriculi }\end{array}$ \\
\hline 1. & $\begin{array}{l}\text { Recht } \\
\text { Link }\end{array}$ & $\begin{array}{l}4,211 \\
4,102\end{array}$ & $\begin{array}{l}4,291 \\
4,282\end{array}$ & $\begin{array}{l}3,777 \\
3,689\end{array}$ & $\begin{array}{l}6,270 \\
6,208\end{array}$ & $\begin{array}{l}7,110 \\
7,112\end{array}$ \\
\hline 2 & $\begin{array}{l}\text { R. } \\
\text { L. }\end{array}$ & $\begin{array}{l}4,503 \\
4,492\end{array}$ & $\begin{array}{l}4,581 \\
4,502\end{array}$ & $\begin{array}{l}4,428 \\
4,409\end{array}$ & $\begin{array}{l}6,642 \\
6,590\end{array}$ & $\begin{array}{l}7,913 \\
7,908\end{array}$ \\
\hline 3. & $\begin{array}{l}\text { R. } \\
\text { L. }\end{array}$ & $\begin{array}{l}5,093 \\
5,010\end{array}$ & $\begin{array}{l}4,927 \\
5,028\end{array}$ & $\begin{array}{l}4,891 \\
4,902\end{array}$ & $\begin{array}{l}6,528 \\
6,486\end{array}$ & $\begin{array}{l}7,012 \\
7,088\end{array}$ \\
\hline 4 & $\begin{array}{l}\text { R. } \\
\mathrm{L} .\end{array}$ & $\begin{array}{l}4,196 \\
4,188\end{array}$ & $\begin{array}{l}4,102 \\
4,281\end{array}$ & $\begin{array}{l}3,982 \\
3,905\end{array}$ & $\begin{array}{l}5,565 \\
5,602\end{array}$ & $\begin{array}{l}6,873 \\
6,794\end{array}$ \\
\hline 5. & $\begin{array}{l}\text { R. } \\
\mathrm{L} .\end{array}$ & $\begin{array}{l}4,820 \\
4,902\end{array}$ & $\begin{array}{l}4,799 \\
4,803\end{array}$ & $\begin{array}{l}4,682 \\
4,659\end{array}$ & $\begin{array}{l}6,827 \\
6,892\end{array}$ & $\begin{array}{l}7,755 \\
7,691\end{array}$ \\
\hline 6. & $\begin{array}{l}\text { R. } \\
\text { L. }\end{array}$ & $\begin{array}{l}4,582 \\
4,599\end{array}$ & $\begin{array}{l}4,528 \\
4,603\end{array}$ & $\begin{array}{l}4,505 \\
4,527\end{array}$ & $\begin{array}{l}6,284 \\
6,302\end{array}$ & $\begin{array}{l}7,303 \\
7,209\end{array}$ \\
\hline 7. & $\begin{array}{l}\text { R. } \\
\text { L. }\end{array}$ & $\begin{array}{l}4,483 \\
4,496\end{array}$ & $\begin{array}{l}4,409 \\
4,381\end{array}$ & $\begin{array}{l}4,194 \\
4,208\end{array}$ & $\begin{array}{l}6,561 \\
6,589\end{array}$ & $\begin{array}{l}7,003 \\
7,098\end{array}$ \\
\hline 8. & $\begin{array}{l}\text { R. } \\
\text { L. }\end{array}$ & $\begin{array}{l}4,071 \\
4,122\end{array}$ & $\begin{array}{l}4,112 \\
4,081\end{array}$ & $\begin{array}{l}3,855 \\
3,791\end{array}$ & $\begin{array}{l}6,018 \\
5,897\end{array}$ & $\begin{array}{l}7,098 \\
7,111\end{array}$ \\
\hline 9. & $\begin{array}{l}\text { R. } \\
\text { L. }\end{array}$ & $\begin{array}{l}4,139 \\
4,092\end{array}$ & $\begin{array}{l}4,281 \\
4,320\end{array}$ & $\begin{array}{l}4,621 \\
4,580\end{array}$ & $\begin{array}{l}6,308 \\
6,328\end{array}$ & $\begin{array}{l}7,235 \\
7,311\end{array}$ \\
\hline 10 & $\begin{array}{l}\text { R. } \\
\text { L. }\end{array}$ & $\begin{array}{l}5,767 \\
5,740\end{array}$ & $\begin{array}{l}5,731 \\
5,697\end{array}$ & $\begin{array}{l}5,518 \\
5,627\end{array}$ & $\begin{array}{l}7,823 \\
7,738\end{array}$ & $\begin{array}{l}9,011 \\
9,131\end{array}$ \\
\hline
\end{tabular}


In Übereinstimmung dazu sind die Stützzellen in der Peripherie kleiner als im Zentrum.

Die Sinneszellen konzentrieren sich mehr im peripheren Teil (siehe Abb. $1 \mathrm{u}$. 2). Zur relativen Verteilung der beiden Zelltypen ergaben sich keine klaren regionale Unterschiede zwischen den zentralen und peripheren Abschnitten der Crista. $45 \%$ der Sinneszellen gehören dem Typ I an. Die Verteilungsrate der Typ I und Typ II Zellen war somit ungefähr gleich in allen drei Cristae des gleichen Innenohres, abgesehen vom Grad individueller Unterschiede von Tier zu Tier. Die Gesamtzahl der Sinneszellen eder Crista des gleichen Tieres wird in der Tabelle gezeigt.

\section{Diskussion}

Die vorliegenden Untersuchungen zeigen, daß zur kontrastreichen Darstellung der Oberflächenmorphologie der Sinnesepithelien des Vestibularapparates, die Silberfärbung (Watanuki 1968, Watanuki et al. 1969, Katagiri et al. 1968) sehr hilfreich ist. Die Succinodehydrogenasereaktion (Barka und Anderson 1964) bringt ausschileßlich die Sinneszellen des Sinnesepitheliums der Crista ampullaris zur Darstellung und ermöglicht somit eine quantitative Auswertung.

Da sich das Sinnesepithel der Sattelform der Crista ampularis anschließt, zeigt die glatte Gesamtoberfläche eine Verkleinerung der Sinneszellen zur Randzone hin an. Lindeman (1969) zeigte den Unterschied der Sinnesepithelien im mittleren und randständigen Teil in Bezug auf Zellgrösse und Zelldichte, und seine Ergebnisse finden nach unseren Untersuchungen unsere volle Bestätigung.

Die von der konkaven, niedrigen Bodenpartie in eine leicht erhöhte Randzone übergehenden Sinneszellen werden von der peripheren Schmalseite der Cupula überdeckt. Die regionalen Unterschiede der Sinnesepithelien könnten damit in Zusammenhang gebracht werden.

Im Sinnesepithelium der Striola sind die Sinneszellen des Typ I größer, mit einer grossen freien Oberfläche und die meisten Zellen vom gemeinsamen Nervenkelch umgeben. Die Succinodehydrogenasereaktivität ist in den großen Sinneszellen der Striola stärker als in den übrigen Sinneszellen, die in und außerhalb der Striola liegen. Ferner wurden stark osmiumhaltige Granula in den Stuitzzellen der Striola gefunden (Lindeman 1969). Dieses metabolische und morphologische Charakteristikum der Striola weist darauf hin, daß sie eine große Funktionsrolle inne hat.

Lindeman (1969) zählte an 5 Innenohren die gesamten Sinneszellen des Sinnesepitheliums im Vestibularorgan aus. Er gab die Durchschnittswerte in der Macula sacculi mit 7,560 Zellen, die der Macula utriculi mit 9,260, Crista anterior mit 5,442, Crista lateralis mit 5,688 und die der Crista posterior mit 5,430 Zellen an. Die Durchschnittsergebnisse dieser vorliegenden Untersuchungen liegen in der Macula sacculi bei 6,472 Zellen, in der Macula utriculi bei 7,439, in der Crista anterior 4,581, Crista lateralis bei 4,587 und in der Cirsta posterior bei 4,438 Zellen. Die Anzahl der Sinneszellen in der Crista anteior und Crista lateralis waren 
fast gleich, während sich in der Crista posterior weniger Zellen fanden als in den übrigen Cristae. Die Sinneszellen der jeweils entsprechenden Crista vom rechten und linken Innenohr, zeigten keine signifikanten Unterschiede in der Zellenanzahl.

Wenn man die vorliegenden Zahlenergebnisse mit denen von Lindeman vergleicht, finden sich bei allen Sinnesepithelien des Verstibularorgans weniger Sinneszellen. Dafür gibt es wahrscheinlich zwei Ursachen:

1) Bei den vorliegenden Untersuchungen wurde die Succino-dehydrogenasereaktion angewandt, die nur die Sinneszellen des Sinnesepitheliums anfärbt. Es ist jedoch nicht ausgeschlossen, daß sich dazwischen einige inaktive Sinneszellen befinden.

2) Die untersuchten Tiere können anderer Abstammung sein.

\section{ZUSAMMENFASSUNG}

Zur morphologischen Oberflächenbetrachtung der Sinnesepithelien des Vestibularapparates wurde die Silberfärbung und Succinodehydrogenasereaktion erfolgreich angewandt.

1) In der Mitte der Crista ampullaris werden oft grössere Sinneszellen gefunden, während an der Peripherie kleinere Zellen angeordnet sind. Die Zelldichte ist an der Peripherie grösser als im Zentrum.

2) Die Striola setzt sich zu zwei Drittel aus Zellen des Typ I zusammen, während ausserhalb der Striola $45 \%$ dem Typ I angehören. Mit 55\% aller Sinneszellen ist der Typ II beteiligt.

3) Die großen Sinneszellen des Typ I, die man in der Striola antrifft, werden gewöhnlich vom gemeinsamen Nervenkelch innerviert.

4) Die großen Sinneszellen der Striola enthalten mehr Succino-dehydrogenaseaktivität als die anderen kleineren, randständigen Zellen der Macula.

Für herzliche Hilfe bei der Druchführung der Arbeit und für die Ubersetzung danken wir bestens Fräulein Otgert Bull.

\section{Literatur}

1) Barka, T. \& Anderson, P.J. Histochemistry. New York, Harper \& Row, 1963.

2) Burlet, H.M. \& Haas, J.H. Die Stellung der Maculae acusticae im Macasus. schädel. Z. Anat. Entwickl.Gesch., 1924, 71, 233-239.

3) Burlet, H.M. \& Hoffmann, J.M. Beitrag zur Kenntnis der Maculae acusticae bei Säugetieren. Arch. Ohr.-, Nas.-, u. Kehlk.-Heilk., 1929, 120, 233-255.

4) Flock, A. Structure of the macula utriculi with special reference to directional interpaly of sensory responses as revealed by morphological polarization. $J$. Cell Biol., 1964, 22, 413-431.

5) Iwata, N. Uber das Labyrinth der Fledermaus mit besonderer Berücksichtigung des statischen Apparates. Aichi J. exp. Med., 1924, 2, 42-172.

6) Katagiri, S., Kawamoto, K. \& Watanuki, K. Some surface views of the stria vascularis and its adjacent areas. Acta oto-laryng. (Stockh.), 1968, 66, 386-389.

7) Katagiri, S., Kawamoto, K., Hori, K. \& Watanuki, K. Some surface views of the inner ear by light microscopy. Acta oto-laryng. (Stockh.), 1968, 66, 493-507. 
8) Lindeman, H.H. Cellular pattern and nerve supply of the vesitbular sensory epithelia. Acta oto-laryng. (Stockh.), 1967, Suppl., 224, 86-95.

9) Lindeman, H.H. Regional differences in structure of the vestibular sensory regions. J. Laryng., 1969, 88, $1-17$.

10) Lindeman, H.H. Studies on the morphology of the sensory regions of the vesitbular apparatus. Ergebn. Anat. Entwickl.-Gesch., 1969, 42, 1, 1-113.

11) Löwenstein, O. \& Wersäll, J. A functional interpretation of the electron microscopic structure of the sensory hairs in the cristae of the elasmobranch Raja clavata in terms of directional sensitivity. Nature (Lond.), 1959, 184, 1807-1815.

12) Löwenstein, O., Osborne, M.P. \& Wersäll, J. Structure and innervation of the stensory epithelia of the labyrinth in the thornback ray (Raja clavata). Proc. roy. Soc. Med., 1964, 160, 1-12.

13) Retzius, G. II. Das Gehörorgan der Reptilien, der Vogel und der Säugestiere. Das Gehörorgan der Wirbeltiere. Samson \& Wallin, Stockholm, 1884.

14) Spoendlin, H.H. Organization of the sensory hairs in the gravity receptors in utricule and saccule of the squirrel monkey. Z. Zellforsch., 1964, 62, 701-716.

15) Spoendlin, H.H. Ultrastructural studies of the labyrinth in squirrel monkeys. The role of the vestibular organs in the exploration of space. NASA SP-77., National Aeronautics \& Space Administration, Washington, 1965, pp. 7-22.

16) Watanuki, K. Sone morphological observations of Reissner's membrane. Acta oto-laryng. (Stockh.), 1968, 66, $40-48$.

17) Watanuki, K., Kawamoto, K. \& Katagiri, S. Repair pattern in the reticular lamina of the organ of Corti after hair cell loss. Ann. Otol. (St. Louis), 1969, 78, 1210-1220.

18) Watanuki, K., Kawamoto, K. \& Katagiri, S. Surface structure of the ampulla of the semicircular canal in the guinea pig. Pract. oto-rhino-laryng. (Basel), 1970, 32, 137-148.

19) Wemer, C.F. Die Differenzierung der Maculae im Labyrinth, insbesondere bei Säugetieren. Z. Anat. Entuickl.Gesch., 1933, 99, 696-709.

20) Wersäll, J. Vestibular receptor cells in fish and mammals. Acta oto-laryng. (Stockh.), 1961., Suppl., 163, 25-29. 